\title{
GENERALIZED SHAPE OPTIMIZATION USING X-FEM AND LEVEL SET METHODS
}

Pierre Duysinx, Laurent Van Miegroet, Thibault Jacobs and Claude Fleury

Automotive Engineering/Multidisciplinary Optimization, Aerospace and Mechanics Department, University of Liège, Chemin des Chevreuils, 1, building B52, 4000 Liège, Belgium

P.Duysinx@ulg.ac.be, L.Vanmiegroet@ulg.ac.be, Th.Jacobs@ulg.ac.be, C.Fleury@ulg.ac.be

\begin{abstract}
This paper presents an intermediate approach between parametric shape optimization and topology optimization. It is based on using the recent Level Set description of the geometry and the novel eXtended Finite Element Method (XFEM). The method takes benefit of the fixed mesh work using X-FEM and of the curves smoothness of the Level Set description. Design variables are shape parameters of basic geometric features. The number of design variables of this formulation is small whereas various global and local constraints can be considered. The Level Set description allows to modify the connectivity of the structure as geometric features can merge or separate from each other. However no new entity can be introduced. A central problem that is investigated here is the sensitivity analysis and the way it can be carried out efficiently. Numerical applications revisit the classical elliptical hole benchmark from shape optimization.
\end{abstract}

Keywords: Shape optimization, topology optimization, X-FEM, level set.

\section{INTRODUCTION}

Topology optimization has experienced an incredible soar since the seminal work of Bendsøe and Kikuchi [2] and is now available within several commercial packages and finite element codes. It is used with great success in industrial applications. Practically, one major advantage of the optimal material distribution formulation is to be able to work on a fixed regular mesh. The drawback is that this formulation comes to very large scale optimization problems, so that one generally considers very simple design problems as the minimum compliance problem with a single volume constraint. Introducing local constraints can lead to very huge problems difficult to handle, whereas controlling geometrical constraints, which are mainly related to manufacturing 
considerations, requires some sophistications. Finally the optimal structure picture needs to be interpreted to construct a parametric CAD model.

Meanwhile, shape optimization, which had received attention since the beginning of the eighties, has been quite unsuccessful in industrial applications. However, shape optimization of internal and external boundaries is of great interest to improve the detailed design of structures against many criteria as restricted displacements, various kinds of stress criteria, buckling, etc. The shape optimization introduces a few design variables since the design problem is formulated on the parameterized CAD model level. The major difficulty is related to the mesh management problems coming from the large shape modifications. Mesh distorsions and Finite Element errors can be reduced using remeshing between two iterations and mesh adaptation tools. However a major technical problem stems also from the sensitivity analysis that requires the calculation of the so-called velocity field. It turns out that shape optimization remains generally quite fragile and delicate to use in industrial context.

In order to circumvent the technical difficulties of the moving mesh problems, a couple of researches have tried to formulate shape optimization with fixed mesh analyses using fictitious domains as in [5], based on fixed grid finite elements in [7] or more recently using projection methods as in [9]. The present work relies on the novel eXtended Finite Element Method (X-FEM) that has been proposed as an alternative to remeshing methods (see [8] or [3] for instance). The X-FEM method is naturally associated with the Level Set [11] description of the geometry to provide a very efficient treatment of difficult problems involving discontinuities and propagations. Up to now the XFEM method has been mostly developed for crack propagation problems [8], but the potential interest of the X-FEM and the level set description for other problems like topology optimization was identified very early in Belytschko et al. [4], Wang et al. [14] or Allaire et al. [1].

The authors see the X-FEM and the Level Set description as an elegant way to fill the gap between topology and shape optimization. The method can be qualified as generalized shape optimization as it has smooth boundary descriptions while allowing topology modifications as holes can merge and disappear. X-FEM enables working on a fixed mesh, as in topology optimization, circumventing the technical difficulties of shape optimization. The structural shape description uses basic level set features (circles, rectangles, etc.) that can be freely combined to generate any shapes. The design variables are parameters of the Level Set features, while constraints can, in principle, be either global (compliance, volume) or local (stress) responses as in shape optimization. A key issue of the problem is the sensitivity analysis. A semi analytical approach has been developed. The work presents clearly validated solutions and still open questions and difficulties. For the numerical applications a complete solution of shape optimization using Level Set description and X-FEM 
has been implemented in the object oriented software, OOFELIE (Open Object Finite Element Lead by Interactive User) [10].

The layout of the paper is thus the following. The Extended Finite Element Method and the Level Set representation are reminded in Sections 2 and 3. Section 4 states the generalized shape optimization problem with X-FEM and the Level Set description. Sensitivity analysis is addressed in Section 5. Finally, in Section 6 an academic applications of shape optimization is reinvestigated to illustrate the proposed extended finite elements and their application to generalized shape optimization.

\section{THE EXTENDED FINITE ELEMENT METHOD}

The eXtended Finite Element Method [3, 8] is a recent method that has been firstly developed for the simulation and the analysis of structures presenting moving boundaries. The main strength of this method is its ability to include discontinuities inside the finite elements. Hence, this method enables to include geometric boundaries, material or phase changes that are not coincident with the mesh.

\subsection{The Basis of the Method}

In order to allow any types of discontinuities inside the elements and therefore to be able to represent discontinuities in the physics fields, it is necessary to add special shape functions to the finite element approximation. For example, in the case of cracked structures, the displacement field is discontinuous and to model the discontinuity, one has to add discontinuous shape functions. The classical finite element approximation used is then extended to embed the discontinuous shape function as in the following equation:

$$
\mathbf{u}(\mathbf{x})=\sum_{i} u_{i} N_{i}(\mathbf{x})+\sum_{j} a_{j} N_{j}(\mathbf{x}) H(\mathbf{x}),
$$

where $N_{i}(\mathbf{x})$ are the classical shape functions associated to degrees of freedom $u_{i}$. The $N_{j}(\mathbf{x}) H(\mathbf{x})$ are the discontinuous shape functions constructed by multiplying a classical $N_{j}(\mathbf{x})$ shape function with a Heaviside function $H(\mathbf{x})$ (presenting a switch value where the discontinuity lies). These extended shape functions are supported only by the enriched (extended) degrees of freedom $a_{j}$. Note that, usually, only the elements near the discontinuity support extended shape functions whereas the other elements remain unchanged. The modification of the displacement field approximation does not introduce a new form of the discretised finite element equilibrium equation but leads to an enlarged problem to solve (see [3] for details):

$$
\mathbf{K} \cdot \mathbf{q}=\mathbf{g} \Leftrightarrow\left[\begin{array}{ll}
K_{u u} & K_{u a} \\
K_{a u} & K_{a a}
\end{array}\right]\left[\begin{array}{l}
u \\
a
\end{array}\right]=\left[\begin{array}{l}
f_{u}^{\mathrm{ext}} \\
f_{a}^{\mathrm{ext}}
\end{array}\right] .
$$


As the elements can now present discontinuous shape functions, the numerical integration scheme has to be modified in order to take care of the discontinuity. In our implementation, the elements embedding a singularity are divided into sub-triangular elements aligned with this discontinuity over which an integration is processed.

\subsection{Representing Holes}

The modeling of material-void interfaces with X-FEM [12] is slightly different from the cracked structure case. For void inclusions and holes, the displacement field is approximated by:

$$
\mathbf{u}(\mathbf{x})=\sum_{i} u_{i} N_{i}(\mathbf{x}) V(\mathbf{x}),
$$

where $V(\mathbf{x})$ takes value ' 1 ' if the node lies inside the material and ' 0 ' otherwise. The elements lying outside the material are removed from the system of equations, whereas the partially filled elements are integrated using the X-FEM integration procedure over solid sub-domain. Modeling holes with the X-FEM is a very appealing method for the shape optimization but also for the topology optimization as no remeshing is needed and no approximation is done on the nature of the voids in opposition to the power penalization of intermediate densities (SIMP) method used in topology optimization.

\section{THE LEVEL SET DESCRIPTION}

The explicit representation of the structural shape of parametric CAD representation forbids deep boundary or topological changes such as creation or fusion of holes. This limitation is one of the main reasons of the low performance generally associated to the shape optimization. Conversely, the Level Set method developed by Sethian [11] which consists of representing the boundary of the structure with an implicit method allows this kind of deep changes.

The Level Set method is a numerical technique first developed to track moving interfaces. It is based upon the idea of representing implicitly the interfaces as a Level Set curve of a higher dimension function $\psi(\mathbf{x}, t)$. The boundaries of the structure is then conventionally represented by the zero level, i.e. $\psi(\mathbf{x}, t)=0$ of this function $\psi$, whereas the filled region is attached to the positive part of the $\psi$ function. In practice, this function is approximated on a fixed mesh by a discrete function which is usually the signed distance function to the curve $\Gamma$ :

$$
\psi(\mathbf{x}, t)= \pm \min _{\mathbf{x}_{\Gamma} \in \Gamma(t)}\left\|\mathbf{x}-\mathbf{x}_{\Gamma}\right\| .
$$

The sign is positive (negative) if $\mathbf{x}$ is inside (outside) the boundary defined by $\Gamma(t)$. Applied to the X-FEM framework, the Level Set is defined on the 
structural mesh and a geometrical degree of freedom representing its Level Set function value is associated at each finite element node. The Level Set is then interpolated on the whole design domain with the classical shape function of the finite element approximation:

$$
\psi(\mathbf{x}, t)=\sum_{i} \psi_{i} N_{i}(\mathbf{x}) .
$$

The combination of different level sets is also one of the appealing characteristic of this method. This property allows easy treatment of merging interfaces and connectivity modifications.

\section{PROBLEM FORMULATION}

The formulation of the optimization problem is similar to a shape optimization problem, but its solution is greatly simplified thanks to the use of the X-FEM and Level Set description.

The geometry and the material layout are specified using Level Sets representations. The user has a library of basic geometric features (in Level Sets) that can be combined to create almost any structural geometry. The available features are circles, ellipsis, squares, triangles, etc. The design variables are chosen among the geometric parameters of these features.

The optimization problem aims at finding the best shape to minimize a given objective function while satisfying mechanical and geometrical design restrictions. The mechanical constraints can either be global responses (e.g. compliance) or local ones as displacements or stress constraints. However, in this preliminary study only static criteria are available.

The number of design variables is generally small as in shape optimization. However the number of constraints may be large if a lot of local stress restrictions, e.g. stress constraints are considered. Nonetheless, large scale problems as in topology optimization are avoided.

The design problem is stated as a general constrained optimization problem:

$$
\begin{array}{cl}
\min & g_{0}(\mathbf{x}) \\
\mathbf{x} & \\
\text { s.t.: } & g_{j}(\mathbf{x}) \leq g_{j}^{\max } \quad j=1 \ldots m \\
& \underline{x}_{i} \leq x_{i} \leq \bar{x}_{i} \quad i=1 \ldots n .
\end{array}
$$

The solution to this problem is carried out using the so-called sequential convex programming. At each iteration, the X-FEM analysis problem is solved and a sensitivity analysis is performed. The solution of the optimization problem is then found by using a CONvex LINearization, CONLIN [6]. The new design point is evaluated and if necessary the procedure is repeated until convergence.

Because of the X-FEM, the geometry has not to coincide with the mesh and the generalized shape optimization problem is carried out on a fixed mesh. This 
circumvents the mesh perturbation problems of classical shape optimization. Sensitivity analysis does not require anymore the velocity field. The present formulation is then, up to a certain point, simpler. However, some technical difficulties can be encountered if a finite difference or a semi-analytical scheme is used for sensitivity analysis as explained in the next section. Basically, the problem is that the perturbation must not change the number of degrees of freedom of the X-FEM approximation.

The Level Set approach is very convenient to modify the geometry because the level sets (and so the holes) can penetrate each other or disappear. Creation of new holes is more problematic since it leads to a non smooth problem. Topological derivatives have then to be used to treat rigorously the problem. This capability is not yet implemented in the present work.

\section{THE SENSITIVITY ANALYSIS METHOD}

As in classical shape optimization, the sensitivity analysis of mechanical responses (such as compliance, displacement, stress ...) is carried out using a semi-analytic approach. In this approach the derivatives of stiffness matrix and load vectors are calculated by finite differences after perturbation of the level set parameter by $\delta x$ :

$$
\frac{\partial \mathbf{K}}{\partial x} \simeq \frac{\mathbf{K}(x+\delta x)-\mathbf{K}(x)}{\delta x} \text { and } \quad \frac{\partial \mathbf{f}}{\partial x} \simeq \frac{\mathbf{f}(x+\delta x)-\mathbf{f}(x)}{\delta x} .
$$

In the classical shape optimization, the computing complexity of the stiffness matrix sensitivity is due to the modifications of the mesh associated to the perturbation $\delta x$ and to the velocity field calculation. In the present X-FEM based approach, one has not to bother with the mesh perturbations as one works on a fixed grid. However, this method exhibits a different drawback with respect to the general shape optimization as the number of elements may vary. The critical situation happens (see Figure 1) when a boundary is very close to a node. Thus, during the perturbation $\delta x$ of the level set, new elements, previously empty, could become partly filled with material and then appear into the formulation. Thus the number of degrees of freedom would change and the dimension of the stiffness matrix would be modified between the level set perturbation.

The strategy that is implemented presently to circumvent the difficulty is the following. As one has only the displacement $\left(u_{i}\right)$ for the elements that are present in the reference configuration, only these elements are taken into account while the contributions coming from the new partly filled elements are ignored. Hence, no new elements are introduced and the size of the stiffness matrix remains unchanged.

This strategy obviously involves an error because it ignores the contributions related to new elements. However, practically the contribution of these 


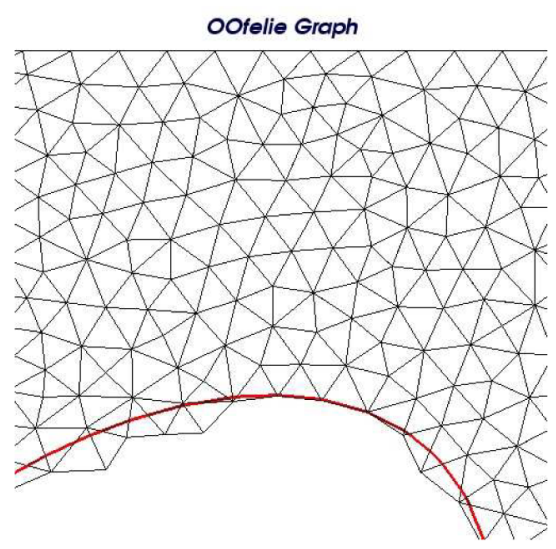

(a) Reference level set and mesh

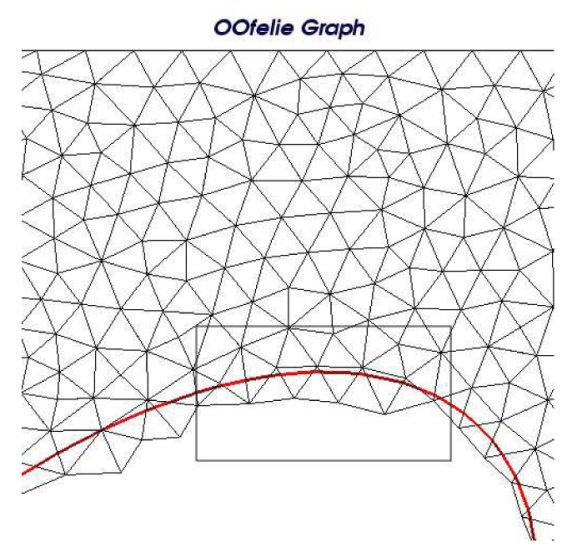

(b) Perturbated level set

Figure 1. Sensitivity difficulty with semi-analytic approach.

elements is so small that the neglected contribution does not alter the precision of the sensitivity. The quality of the approximation is illustrated in the application section with the elliptical hole problem.

Of course the ultimate solution to the problem should resort to a fully analytical sensitivity of the stiffness matrix, but this would be rather restrictive for industrial applications. On-going work is devoted to investigate two kinds of other strategies to reduce the error of the semi-analytic approach:

(1) One can keep a narrow band (boundary layer) of elements with very soft mechanical properties around the level set $\psi=0$ in order to prevent the variation of the total number of degrees of freedom.

(2) One could define a tolerance zone around the Level Set. If the discontinuity in an element lies inside this zone, add the connected elements to the set of cut ones.

These two alternative methods have the advantage of keeping the number of degrees of freedom constant and then they do not create or remove elements during the perturbation step. Hence, the computation of the sensitivity would lead to a more accurate result as all elements are taken into account in the perturbated stiffness matrix. However, the presence of these elements will probably introduce a dependency upon the mechanical properties associated to the narrow softening elements band like in topology optimization with the power $p$ coefficient in the SIMP law. Moreover, the use of this two methods does not take fully advantage of the X-FEM as we re-introduce an approximation of the void as a weak material. 


\section{APPLICATIONS}

\subsection{Implementation}

The X-FEM method and its Level Set description have been implemented in an object oriented $(\mathrm{C}++)$ multiphysics finite element code, OOFELIE that is commercialized by Open Engineering [10].

In OOFELIE, any mechanical result can be chosen as objective functions and constraints that is: compliance and potential energy, all stress components, displacements and geometric results. However in this study, solely compliance minimization is used. Implementation of the X-FEM method is available in 2-D problems with a library of both quadrangle and triangle elements. The CONLIN optimizer by Fleury [6] has also been coupled in the OOFELIE environment and an optimization framework has been created.

\subsection{Plate with an Elliptical Hole}

The plate with a hole is a classical benchmark from shape optimization. To remind the reader, a large plate with a hole in the middle is subjected to a biaxial stress field. The goal of the optimization problem is to find the optimal shape to minimize the compliance of the structure with a constraint on the total volume of the hole. From the analytical solution, we know that the solution is an elliptical hole aligned with the principal stresses. Figure 2 left shows the quarter of the initial design domain, an elliptic hole with a $45^{\circ}$ orientation.

Here the particular values are considered. The dimensions of the plate are $2 \times 2 \times 1 \mathrm{~m}$. The domain is covered with a transfinite mesh with 30 nodes on each side. The applied biaxial stress field is $\sigma_{x}=2 \sigma_{0}$ and $\sigma_{y}=\sigma_{0}$ and the material properties associated are: Young modulus $E=1 \mathrm{~N} / \mathrm{m}^{2}$, Poisson's

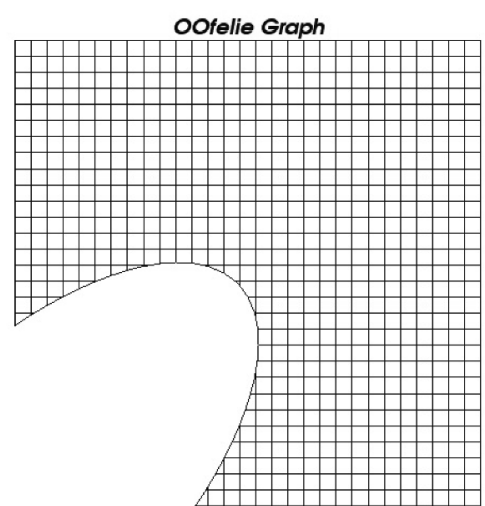

(a) Initial geometry

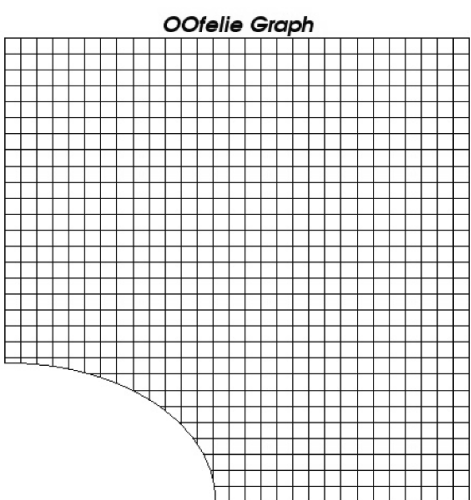

(b) Final geometry

Figure 2. Plate with a hole. 
Table 1. Validation of semi-analytical sensitivity analysis approximation.

\begin{tabular}{lccc}
\hline Design variables & Finite differences & Semi-analytical approach & Relative error (\%) \\
\hline$a=0.6$ & 3698.0000 & 3691.3344 & 0,1802 \\
$\theta=\pi / 4$ & 478.0000 & 477.0641 & 0.1957 \\
\hline$a=0.6$ & 783.8000 & 781.3920 & 0.3072 \\
$\theta=0$ & 11.6239 & 11.6235 & 0.0029 \\
\hline
\end{tabular}

ration $v=0.3$. The plane stress state is assumed. The variables are the angle $\theta$ and the long axis $a$.

Three iterations with CONLIN optimizer are necessary to come to the solution, an ellipsis aligned with the principle stresses (see Figure 2(b)).

Let us remark the discretization of the geometry using the level set. The boundaries are represented using the linear finite element shape functions, so that the boundary is approximated using piecewise linear segments. This can lead to discretization errors of the geometry as noted in [13].

The elliptical hole serves also to validate the approximated semi-analytical sensitivity analysis that has been proposed in Section 5. Table 1 gives the sensitivities of compliance calculated by finite differences and semi-analytical approach for different combination of the design variables $a$ and $\theta$. The results were obtained with a relative perturbation of the design variables of $\delta=10^{-4}$. The results show the quality of the proposed semi-analytical approximation.

\section{CONCLUSION}

An intermediate approach between shape and topology optimization has been developed using extended finite elements and Level Set description. The method combines the advantage of the fixed mesh approach of topology optimization and the smooth curve description of shape optimization. Obtained results show that this new approach is promising and deserve further efforts.

The investigation of a semi-analytic sensitivity analysis with X-FEM and Level Set is an original contribution of the paper. The problem of elements becoming partially filled has been identified and a first strategy to circumvent the problem has been validated. On-going work explores other alternative approaches.

The solution of 2-D problems is presently available. Future work is devoted to attack 3-D problems, dynamic problems, and multiphysic (electromechanical) problems. 


\section{ACKNOWLEDGMENTS}

Part of this work has been realized in the framework of project ARC MEMS, Action de recherche concertée 03/08-298 funded by the Communauté Française de Belgique and by project RW 02/1/5183, MOMIOP funded by the Walloon Region of Belgium.

\section{REFERENCES}

[1] Allaire, G., Jouve, F. and Toader, A.M., Structural optimization using sensitivity analysis and a level-set method, Journal of Computational Physics, 194(1), 363-393 (2004).

[2] Bendsøe, M.P. and Kikuchi, N., Generating optimal topologies in structural design using a homogenization method, Computer Methods in Applied Mechanics and Engineering, 71, 197-224 (1988).

[3] Belytschko, T., Parimi, C., Moes, N., Sukumar, N. and Usui, S., Structured extended finite element methods for solids defined by implicit surfaces, International Journal for Numerical Methods in Engineering, 56, 609-635 (2003).

[4] Belytschko, T., Xiao, S. and Parimi, C., Topology optimization with implicit functions and regularization, International Journal for Numerical Methods in Engineering, 57, 1177-1196 (2003).

[5] Dankova and Haslinger, J., Numerical realization of a fictitious domain approach used in shape optimization. Part I. Distributed controls, Applications of Mathematics, 41(2), 123-147 (1996).

[6] Fleury, C., CONLIN: An efficient dual optimizer based on convex approximation concepts, Structural Optimization, 1, 81-89 (1989).

[7] Garcia-Ruiz, M.J. and Steven, G.P., Fixed grid finite element in elasticity optimization Engineering Computations, 16(2), 145-164 (1999).

[8] Moes, N., Dolbow, J., Sukumar, N. and Belytschko, T., A Finite Element Method for Crack Growth without Remeshing, International Journal for Numerical Methods in Engineering, 46, 131-150 (1999).

[9] Norato, J., Haber, R., Tortorelli, D. and Bendsøe, M.P., A geometry projection method for shape optimization, International Journal for Numerical Methods in Engineering, 60(14), 2289-2312 (2004).

[10] OOFELIE, An Object Oriented Finite Element Code Led by Interactive Execution, Internet resources: www.open-engineering.com.

[11] Sethian, J., Level Set Methods and Fast Marching Methods: Evolving Interfaces in Computational Geometry, Fluid Mechanics, Computer Vision and Materials Science, Cambridge University Press (1999).

[12] Sukumar, N., Chopp, D.L., Noës, N. and Belytschko, T., Modelling holes and inclusions by Level Set in the extended finite element method, Computer Methods in Applied Mechanics and Engineering, 190, 6183-6200 (2001).

[13] Van Miegroet, L., Moes, N., Fleury, C. and Duysinx, P., Generalized shape optimization based on the level set method, in Proceedings of the 6th World Congress of Structural and Multidisciplinary Optimization, J. Herskowitz (ed.), Rio de Janeiro, Brazil, May 30-June 3 (2005).

[14] Wang, M., Wang, X. and Guo, D., A level set method structural topology optimization, Computer Methods in Applied Mechanics and Engineering, 191, 227-246 (2003). 